\title{
Phenotype selection for detecting variable genes: a survey of cardiovascular quantitative traits and TNF locus polymorphism
}

\author{
Mun-Gwan Hong ${ }^{1}$, Anna M Bennet ${ }^{2,3}$, Ulf de Faire ${ }^{3,4}$ and Jonathan A Prince ${ }^{\star, 1}$
}

\begin{abstract}
${ }^{1}$ Department of Cell and Molecular Biology, Karolinska Institute, Stockholm, Sweden; ${ }^{2}$ Department of Public Health and Primary Care, University of Cambridge, Cambridge, UK; ${ }^{3}$ Division of Cardiovascular Epidemiology, Institute of Environmental Medicine, Karolinska Institute, Stockholm, Sweden and ${ }^{4}$ Department of Cardiology, Karolinska Hospital, Karolinska Institute, Stockholm, Sweden
\end{abstract}

The practice of using discrete clinical diagnoses in genetic association studies has seldom led to a replicable genetic model. If, as the literature suggests, weak genotype-phenotype relationships are detected when clinical diagnoses are used, power might be increased by exploring more fundamental biological traits. Emerging solutions to this include directly modeling levels of the protein product of a gene (usually in plasma) and sequence variation specifically in/around that gene, as well as exploring multiple quantitative traits related to a disease of interest. Here, we attempt a strategy based upon these premises examining sequence variants near the TNF locus, a region widely studied in cardiovascular disease. Multilocus genotype models were used to perform a systematic screen of 18 metabolic and anthropometric traits for genetic association. While there was no evidence for an effect of TNF polymorphism on plasma TNF levels, a relatively strong effect on plasma PAI-1 levels did emerge $(P=0.000019)$, but this was only evident in post-myocardial infarction patients. Modeled jointly with the common 4G/5G insertion/deletion polymorphism of SERPINE1 (formerly PAI), this effect appears large (10\% of variance explained versus $2 \%$ for SERPINE1 4G/5G). We exhibit this finding cautiously, and use it to illustrate how transitioning the study of disease risk to quantitative traits might empower the identification of functionally variable genes.

Further, a case is highlighted where association between sequence variation in a gene and its product is not readily apparent even in large samples, but where association with a down-stream pathway may be. European Journal of Human Genetics (2007) 15, 685-693. doi:10.1038/sj.ejhg.5201803; published online 14 March 2007

Keywords: TNFA; polymorphism; PAI-1

\section{Introduction}

Non-replication of genetic association studies is extensive. ${ }^{1,2}$ The vast majority of such studies entail the examination of a single discretely defined disease state in

\footnotetext{
*Correspondence: Dr JA Prince, Department of Cell and Molecular Biology, Karolinska Institute, Berzelius väg 35, 17177 Stockholm, Sweden. Tel: + 468524 86274; Fax: + 468324 826; E-mail: Jonathan.Prince@ki.se

Received 12 May 2006; revised 12 October 2006; accepted 10 January 2007; published online 14 March 2007
}

case-control and/or family-based models. The field is dominated by an approach where many genes (typically across an equal number of publications) are examined in relation to a single disease phenotype that is deemed important. At the extreme, whole genome association studies are now being planned and executed, typically with a single target phenotype. ${ }^{3,4}$ The focus on disease is natural, given the importance its resolution has to humanity, but directly tackling the questions of its genetic etiology may be overzealous until we understand more about the functional characteristics of gene 
polymorphism. ${ }^{5}$ Thus, it may be prudent to invert the question from 'is there a gene affecting my phenotype?' to 'what phenotype may allow me to find a genetic effect at all?'.

In contrast to studies directly modeling disease risk, some of the strongest and most reliable associations that have emerged involve quantitative phenotypes that have been obtained without the inherent complexities of clinical diagnosis. One of the best examples is the effect of variation in the angiotensin converting enzyme gene (ACE) upon plasma levels of the ACE protein. ${ }^{6,7}$ The effect is overwhelming, with as much as $50 \%$ of the variance in plasma ACE levels explained by polymorphism at the ACE locus. ${ }^{6}$ This is illustrative of what can happen when one selects an appropriate phenotype to detect underlying genetic variability. The strength of the effect also allows for a more clear dissection of a genetic model that best fits the data. Consequently, the evidence is robust that there is allelic heterogeneity (multiple functional sites in a gene) that influence the ACE trait, something that can be seen clearly given the strong genotype-phenotype relationship. ${ }^{7}$ In contrast, if weak associations are evident for disease risk, then the reliability of the genetic model is going to be equally suspect. Other excellent examples include the strong effect of dopamine $\beta$-hydroxylase gene $(D B H)$ polymorphism on plasma DBH levels ${ }^{8}$ an effect of histidine rich glycoprotein $(H R G)$ polymorphism on plasma HRG levels ${ }^{9}$ and an association between $C R P$ variation and plasma CRP levels. ${ }^{10}$

Despite evidence for weak effects on disease, some genes have enjoyed so much study that there is reasonable cumulative evidence that functional variation occurs in a region, even if the nature of that variation remains a mystery. One such gene encodes tumor necrosis factoralpha $(T N F-\alpha)$, which has been the subject of perhaps more than 1000 genetic association studies (as returned from PubMed using the search string 'tnfa* or tnf-alpha, gene*, polymorph*, assoc*'). A cursory scan of those studies illustrates some of the primary problems of genetic association studies. Most have only examined a single common promoter variant $(-308 \mathrm{~A} / \mathrm{G})$, most are based upon small samples (a few hundred individuals), and the majority assesses a single disease or other clinical end point. There are, however, examples of well-powered studies implicating the TNF region, in particular in myocardial infarction, ${ }^{3}$ but this too has been followed by non-replication. ${ }^{11}$ A previous study examining TNF variation in a large coronary heart disease (CHD) sample provided only weak evidence of an effect upon TNF plasma levels (in contrast to the above cited examples of ACE etc). ${ }^{12}$ Against this background, we have performed a systematic evaluation of quantitative traits typically examined in cardiovascular disease, providing a broader search for phenotypes that may be able to detect the presence of functional variation within the TNF region.

\section{Materials and methods Clinical materials}

Patient DNA samples employed in this study derive from individuals participating in the Stockholm Heart Epidemiology Program (SHEEP), a population-based casecontrol study aimed at investigating the effects of various risk factors for myocardial infarction in men and women. ${ }^{13}$

It consists of Swedish men and women aged 45-70 who were admitted to hospitals for treatment of first-time myocardial infarction (MI) events and population-based controls without a history of coronary disease. The present study is based on cases that survived 28 days after their first MI and had no further events before blood sampling, and their corresponding, frequency-matched controls who were randomly selected from the Stockholm County population registry after stratification for age (in 5-year intervals), sex and residential area. Five control candidates per case were sampled at the case incidence so that nonrespondent controls could be replaced by another candidate who belonged to the same study base as the index case. Criteria for myocardial infarction were (i) certain symptoms according to case history information, (ii) specified changes in blood levels of the enzymes CK and LD and (iii) specified ECG changes, according to criteria accepted by the Swedish Association of Cardiologists in 1991. Participants were invited to a physical examination that included blood sampling after an overnight fast, approximately 3 months after the MI event to allow for a stable metabolic period. The ethics committee at the Karolinska Institute approved the study and all participants gave informed consent. A detailed description of these individuals and the examined traits can be found in Table 1.

\section{Blood chemistry}

Blood samples were collected approximately 3 months after the MI event. The samples were separated within $1 \mathrm{~h}$ and kept frozen until analysis. DNA was isolated using RapidPrep Macro Genomic DNA isolation kit (Pharmacia Biotech, Sweden). Blood lipid levels were determined using routine methods at the different hospitals using enzymatic colorimetric assays on fully automated equipment. Body mass index was calculated as $\mathrm{kg} / \mathrm{m}^{2}$ based on anthropometric data from each participant obtained at the health examination. The mean of two blood pressure measurements were used to calculate systolic and diastolic blood pressure. The assays used to measure fibrinogen, PAI-1, fasting glucose, IGFBP-1, CRP, IL- 6 , TNF- $\alpha$ have been described elsewhere. ${ }^{12,14-16}$

\section{SNP selection and verification}

Five SNPs from the TNF promoter region were selected in this study (from $5^{\prime}$ to $3^{\prime}$ of the gene and from pter to qter along the chromosome) rs1799964 (-1031T/C), rs1800630 $(-863 \mathrm{C} / \mathrm{A}), \mathrm{rs} 1799724(-857 \mathrm{C} / \mathrm{T}), \mathrm{rs} 1800629(-308 \mathrm{G} / \mathrm{A})$, 
Table 1 Distribution of selected traits in Ml cases and controls

\begin{tabular}{|c|c|c|c|c|c|c|c|c|}
\hline \multirow[b]{2}{*}{ Trait } & \multicolumn{4}{|c|}{ Men } & \multicolumn{4}{|c|}{ Women } \\
\hline & Controls & $\mathrm{N}$ & Cases & $\mathrm{N}$ & Controls & $\mathrm{N}$ & Cases & $\mathrm{N}$ \\
\hline ge (years) & $58.8 \pm 0.22$ & 1054 & $58.3 \pm 0.24$ & 852 & $61.9 \pm 0.30$ & 507 & $61.5 \pm 0.36$ & 357 \\
\hline ody mass index $\mathrm{kg} / \mathrm{m}^{2}$ ) & & 702 & $26.5 \pm 0.16$ & & 0.20 & & $26.7 \pm 0.33$ & 236 \\
\hline DBP (Diastolic blood press & $84.7 \pm 0.40$ & 700 & $80.9 \pm .0 .45$ & 50 & $81.9 \pm 0.51$ & 507 & \pm 0.66 & 236 \\
\hline SBP (Systolic blood pressure mm Hg) & $139.4 \pm 0.78$ & 700 & $132.0 \pm 0.91$ & 508 & $142.6 \pm 1.10$ & 393 & $131.5 \pm 1.45$ & 236 \\
\hline Ins (Insulin mU/ml) & $10.6 \pm 0.29$ & 754 & $14.4 \pm 0.61$ & 602 & $9.9 \pm 0.38$ & 414 & $14.4 \pm 0.85$ & 297 \\
\hline $\begin{array}{l}\text { HOMA* }^{*} \text { (Homoeostasis model } \\
\text { assessment) }\end{array}$ & $2.63 \pm 0.092$ & 685 & $3.89 \pm 0.23$ & 53 & $2.55 \pm 0.18$ & 378 & $3.90 \pm 0.28$ & 269 \\
\hline $\begin{array}{l}\text { Gluc (Glucose } \mathrm{mg} / \mathrm{dl} \text { ) } \\
\text { IGFBP1 (Insulin-like growth factor binding } \\
\text { protein } 1 \mathrm{mg} / \mathrm{l} \text { ) }\end{array}$ & $\begin{array}{l}5.44 \pm 0.053 \\
26.1 \pm 0.74\end{array}$ & $\begin{array}{l}951 \\
755\end{array}$ & $\begin{array}{l}5.92 \pm 0.077 \\
26.6 \pm 0.88\end{array}$ & $\begin{array}{l}752 \\
602\end{array}$ & $\begin{array}{l}5.24 \pm 0.070 \\
33.3 \pm 1.02\end{array}$ & $\begin{array}{l}466 \\
414\end{array}$ & $\begin{array}{l}5.79 \pm 0.12 \\
37.5 \pm 1.67\end{array}$ & $\begin{array}{l}329 \\
299\end{array}$ \\
\hline FB (Fibrinogen $\mathrm{g} / \mathrm{l}$ ) & $3.43 \pm 0.023$ & 992 & $3.65 \pm 0.030$ & 789 & $3.62 \pm 0.034$ & 467 & $3.94 \pm 0.052$ & 341 \\
\hline protein mg/l) & & 735 & 3.7 & & & & 4 & 295 \\
\hline IL6 (Interleukin-6 ng/l) & 4.7 & 590 & $8.98 \pm 1.40$ & & 6.4 & $2 \varepsilon$ & 1.22 & 275 \\
\hline TNF- $\alpha$ (Tumor necrosis factor ng/l) & $2.17 \pm$ & 636 & $2.39 \pm 0.090$ & 5 & 0.094 & 374 & 0.124 & 280 \\
\hline $\begin{array}{l}\text { PAl-1 (Plasminogen activator inhibitor- } \\
1 \mathrm{mg} / \mathrm{l} \text { ) }\end{array}$ & & 1007 & $20.4 \pm 0.69$ & 80 & & 470 & $20.5 \pm 0.94$ & 341 \\
\hline $\begin{array}{l}\text { HDL-c (High density lipoprotein } \\
\text { cholesterol } \mathrm{mmol} / \mathrm{l} \text { ) }\end{array}$ & $1.19 \pm 0.010$ & 1046 & $1.02 \pm 0.009$ & 842 & $1.49 \pm 0.016$ & 503 & $1.23 \pm 0.018$ & 353 \\
\hline $\begin{array}{l}\text { LDL-c (Low density lipoprotein } \\
\text { cholesterol } \mathrm{mmol} / \mathrm{l} \text { ) }\end{array}$ & $3.92 \pm 0.028$ & 1041 & $4.17 \pm 0.033$ & 822 & $4.05 \pm 0.047$ & 503 & $4.36 \pm 0.059$ & 351 \\
\hline TC (Total cholesterol mmol/l) & $5.81 \pm$ & 1053 & $6.08 \pm 0.038$ & 84 & $6.15 \pm$ & 50 & $6.47 \pm 0.0$ & 358 \\
\hline TG (Triglyceride $\mathrm{mmol} / \mathrm{l}$ ) & & 1053 & & & & & $2.02 \pm 0.0$ & 359 \\
\hline APOA1 (Apolipoprotein A1 g/l) & & 1051 & & 8 & & & & 357 \\
\hline APOB (Apolipoprotein B $\mathrm{g} / \mathrm{l}$ ) & $1.47 \pm 0.011$ & 1051 & $1.60 \pm 0.012$ & 848 & $1.52 \pm 0.018$ & 505 & $1.73 \pm 0.024$ & 357 \\
\hline
\end{tabular}

Anthropometric and metabolic traits (units) of clinical materials stratified by gender and disease. (values are mean \pm SEM). * Calculated as [fasting glucose $(\mathrm{mg} / \mathrm{dl}) \times$ fasting insulin $(\mathrm{nU} / \mathrm{l})] / 22.5$.

and rs3615525 (-238G/A), details on which may be found in the dbSNP database (http://www.ncbi.nlm.nih.gov/SNP/) under their respective IDs. Surrounding $50 \mathrm{bp}$ sequences in each direction were examined for repeats and duplicated sequences using Repeat-masker (http://repeatmasker.genome. washington.edu/cgi-bin/RepeatMasker/) and Blast (http:// www.ncbi.nlm.nih.gov/blast). To verify that SNPs were polymorphic in our study populations, each SNP was tested in a set of 16 Swedish control samples.

\section{Genotyping}

Genotyping of SNPs was performed using dynamic allelespecific hybridization. ${ }^{17}$ All PCR reactions were run in $10-20 \mu \mathrm{l}$ volumes with $1.5 \mathrm{mM} \mathrm{MgCl}_{2}$ and using 5-20 ng genomic DNA.

\section{Statistical analysis}

Deviation from Hardy-Weinberg equilibrium for genotypes at individual loci as well as differences in haplotype and diplotype distributions between discrete groups were assessed using the $\chi^{2}$ statistic. Correlations between traits were established using simple linear regression. Deviation from normality for trait distributions was assessed using a Kolgomorov-Smirnov test. Tests for association between genotypes and quantitative traits were performed using analysis of variance (ANOVA) with post hoc tests being conducted with Fisher's protected least significant differences (PLSD) test. As the sample was large, all initial ANOVA analyses were performed on untransformed data. Effects with the greatest magnitude in the event of a nonnormally distributed trait were also modeled using the Mann-Whitney $U$-test. The above statistical analyses were performed using StatView version 5.0 (Abacus Concepts). Haplotype frequencies and diplotype configurations were estimated using PHASE v2.1. ${ }^{18}$ LD between marker pairs was estimated using the $r^{2}$ metric. ${ }^{19}$

\section{Results}

We limited our initial investigation to the 18 phenotypes that are traditionally studied in relation to cardiovascular disease. $^{20,21}$ While many of these exhibit strong correlations, we opted to investigate each independently recognizing that there would be some redundancy. The intention was to perform a systematic evaluation of traits without prior beliefs about which systems might be affected by TNF variation, other than that they might be CHD-related. For the genetic markers tested, there was no evidence of deviation from Hardy-Weinberg equilibrium.

An important consideration in our initial screen was how to compress genotypes into a single manageable genetic 
Table 2 Common haplotypes extending across the TNF promoter

\begin{tabular}{lclllll}
\hline Name & Count (freq.) & rs1799964 & rs1800630 & rs1799724 & rs1800629 & rs3615525 \\
\hline H1 & $2997(54.5 \%)$ & T & C & C & G & G \\
H2 & $911(16.5 \%)$ & C & A & C & C & A \\
H3 & $881(16.0 \%)$ & T & C & T & G & G \\
H4 & $429(7.8 \%)$ & T & C & C & G & A \\
H5 & $172(3.1 \%)$ & C & C & C & G \\
H6 & $79(1.4 \%)$ & C & A & T & G \\
H7 & $30(0.5 \%)$ & T & & & \\
\hline
\end{tabular}

Detected haplotypes that have been estimated using PHASE v2.1. Four haplotypes with counts $<10$ are not shown. The two markers used for diplotype reconstruction are shaded.

factor. With five markers and 18 traits, the number of tests becomes relatively large. Towards this end, we identified two of the five single markers that delineate the most common (>10\%) haplotypes (Table 2) and estimated diplotype configurations of those common haplotypes for each individual (see $\mathrm{Gu}$ et $a .^{22}$ for details and an application of this strategy). We used PHASE v2.1 to arrive at the probable configurations using rs1800630 and rs1800629 and noted that the configurations based upon these two markers are essentially phase known (near 100\% probability). This occurs since the 'A' allele at rs1800630 and the ' $A$ ' allele at rs1800629 never occur together (Table 2), indicating that only three of the four possible haplotypes exist at detectable frequencies for these two markers . For practical reference, we point out that this strategy is equivalent to what has been done for years in the case of $A P O E$, using two markers to distinguish all diplotype configurations of e2, e3, and e4 alleles (eg Strittmatter et al. ${ }^{23}$ ).

We tested diplotype-phenotype relationships initially in the whole population using ANOVA, adjusting for age, gender, and disease status in the model. We recognized that interactions with these factors might be present, but acknowledged that they would probably be easier to consider post hoc if any significant findings were made in the primary analyses. The results of this test are shown in Table 3. Three significant findings were made for CRP $\left(\mathrm{F}_{5,1966}=2.7, P=0.019\right)$, HOMA $\left(\mathrm{F}_{5,1791}=2.6, P=0.023\right)$, and PAI- $1\left(\mathrm{~F}_{5,2522}=4.4, P=0.0005\right)$. Of note, there was no evidence for an effect of TNF variation on plasma TNF- $\alpha$ protein levels. We focussed on the PAI-1 finding, as it withstands strict Bonferroni correction for multiple testing (considering 18 initial ANOVA omnibus tests). We then tested for possible interactions of diplotype with age, gender, or disease status in a second-order factorial ANOVA model. The disease by diplotype interaction term emerged as significant $\left(\mathrm{F}_{5,2522}=3.2, P=0.0065\right)$ and so stratified models were also tested to investigate this more closely. An effect was only evident in the disease group $\left(\mathrm{F}_{5,1087}=6.0\right.$, $P=0.000019)$, in contrast to $\left(\mathrm{F}_{5,1425}=0.2, P=0.93\right)$ incontrols (Figure 1). We estimated from this that variation at the TNF locus contributes to approximately $10 \%$ of the variance in plasma PAI-1 levels in this group. For comparison, we also obtained SERPINE1 locus genotypes (the common $4 \mathrm{G} / 5 \mathrm{G}$ variant) and included it as a factor in ANOVA models. This factor was also significant in the disease group $\left(\mathrm{F}_{2,1073}=4.5, P=0.012\right)$ but not in the controls. We estimated that SERPINE1 genotype accounted for approximately $2 \%$ of variation in PAI- 1 plasma levels. Quantitatively, the greatest difference in PAI-1 levels from pair-wise diplotype comparisons was between the $\mathrm{H} 2 \mathrm{H} 2$ and $\mathrm{H} 2 \mathrm{H} 3$ categories (Figure 1). We note that this comparison is highly significant based upon non-parametric analysis (Mann-Whitney $U$-test) $(Z=3.7$, $P=0.0002$; compared with $P=0.0011$ for a simple $t$-test on un-transformed data). As the diplotype strategy used above compresses some of the rarer haplotypes, models were also tested using all the five markers for the PAI-1 phenotype. The results of this are shown in Figure 2. Finally, the 2-locus diplotype model was also tested for CRP and HOMA in cases and controls separately (since these traits showed borderline significance in the initial screen). Results for this analysis were negative (for CRP; $\mathrm{F}_{5,1108}=1.3, P=0.24$ and $\mathrm{F}_{5,830}=1.6, P=0.15$ in controls and cases respectively; for HOMA, $\mathrm{F}_{5,1015}=1.7, P=0.15$ and $\mathrm{F}_{5,748}=1.2, P=0.32$ in controls and cases, respectively).

Based upon the maximum effect being observed upon PAI-1 levels, and following from the broader search using diplotype models, we also explored for independent marker effects. To do this, we fitted first-order factorial ANOVA models with each of the markers in a step-wise forward selection algorithm. The value of doing this is to capture potential allelic heterogeneity that may be acting in the region (i.e. two or more sites that independently affect a phenotype) (e.g. Cox et al. ${ }^{7}$ ). The unusual model that emerged from the diplotype strategy indicated that addressing this might be important. The results of this analysis are shown in Table 4.

Both 2- and 5-locus diplotype models were also used to test for association with case-control status, the specific goal for which this sample was originally acquired. ${ }^{13}$ There was no evidence that diplotype group frequencies differed between cases and controls (not shown). 
Table 3 Evaluation of TNF locus diplotypes with quantitative traits

\begin{tabular}{|c|c|c|c|c|c|c|c|}
\hline Trait & $\mathrm{H} 1 \mathrm{H} 1$ & $\mathrm{H} 1 \mathrm{H} 2$ & $\mathrm{H} 1 \mathrm{H3}$ & $\mathrm{H} 2 \mathrm{H} 2$ & $\mathrm{H} 2 \mathrm{H} 3$ & $\mathrm{H} 3 \mathrm{H} 3$ & Significance \\
\hline POA1 & $1.47 \pm 0.008(1102)$ & $1.48 \pm 0.011(652)$ & $1.46 \pm 0.011(623)$ & $1.49 \pm 0.035(79)$ & $1.46 \pm 0.023(14$ & $1.51 \pm 0.037(71$ & $F(5,2658)=0.9, P=0.49$ \\
\hline POB & $1.55 \pm 0.012(1102)$ & $1.57 \pm 0.016(652)$ & $1.55 \pm 0.015(623)$ & $1.49 \pm 0.039(79)$ & $1.54 \pm 0.038(143)$ & $1.54 \pm 0.04(71)$ & $\mathrm{F}(5,2648)=0.9, P=0.48$ \\
\hline BMI & $25.9 \pm 0.14(741)$ & $25.8 \pm 0.18(436)$ & $26.2 \pm 0.18(413)$ & $26.2 \pm 0.55(48)$ & $26.3 \pm 0.45(102)$ & $26.4 \pm 0.57(38)$ & $F(5,1766)=0.7, P=0.64$ \\
\hline DBP & $81.7 \pm 0.39(738)$ & $82.3 \pm 0.53(436)$ & $82.2 \pm 0.51(412)$ & $80.2 \pm 1.26(48)$ & $82.6 \pm 1.13(102)$ & $83 \pm 1.44(38)$ & $F(5,1762)=0.6, P=0.72$ \\
\hline $\mathrm{FB}$ & $3.59 \pm 0.025(1028)$ & $3.62 \pm 0.035(608)$ & $3.59 \pm 0.033(588)$ & $3.59 \pm 0.085(74)$ & $3.62 \pm 0.067(137)$ & $3.56 \pm 0.102(66)$ & $\mathrm{F}(5,2489)=0.2, P=0.97$ \\
\hline Gluc & $5.53 \pm 0.05(994)$ & $5.6 \pm 0.08(584)$ & $5.64 \pm 0.09(568)$ & $5.6 \pm 0.22(70)$ & $6.01 \pm 0.24(133)$ & $5.47 \pm 0.18(65)$ & $\mathrm{F}(5,2402)=1.7, P=0.13$ \\
\hline HDL-c & $1.2 \pm 0.011(1096)$ & $1.22 \pm 0.014(646)$ & $1.17 \pm 0.014$ (619) & $1.23 \pm 0.043(79)$ & $1.2 \pm 0.033(142)$ & $1.21 \pm 0.046(70)$ & $\mathrm{F}(5,2640)=1.7, P=0.14$ \\
\hline HOMA & $3 \pm 0.11(740)$ & $3.04 \pm 0.16(448)$ & $3.25 \pm 0.25(418)$ & $2.53 \pm 0.25(51)$ & $4.33 \pm 0.61(107)$ & $3.3 \pm 0.62(39)$ & $\mathrm{F}(5,1791)=2.6, P=\mathbf{0 . 0 2 3}$ \\
\hline IL6 & $6.71 \pm 0.97(686)$ & $5.24 \pm 0.92(418)$ & $6.99 \pm 1.29(375)$ & $8.23 \pm 4.36(46)$ & $7.39 \pm 3.27(90)$ & $12.25 \pm 7.11(34)$ & $\mathrm{F}(5,1637)=0.7, P=0.61$ \\
\hline & $11.9 \pm 0.35(822)$ & $11.9 \pm 0.42(501)$ & $11.9 \pm 0.47(460)$ & $13.9 \pm 3.99(55)$ & $13.4 \pm 1.13(116)$ & $12.9 \pm 1.47(44)$ & $F(5,1986)=0.9, P=0.47$ \\
\hline LDL-C & $4.07 \pm 0.0$ & $4.11 \pm 0.041(639)$ & $4.07 \pm 0.038(611)$ & $4 \pm 0.094$ & $.093(140)$ & $4.2 \pm 0.12(70)$ & $\mathrm{F}(5,2613)=1.3, P=0.26$ \\
\hline PAl-1 & $17.8 \pm 0.5(1042)$ & $16.5 \pm 0.6(615)$ & $17.2 \pm 0.7(595)$ & $14.4 \pm 1.7(75)$ & $22.6 \pm 2(140)$ & $16.3 \pm 1.8(67)$ & $\mathrm{F}(5,2522)=4.4, P=0.0005$ \\
\hline SBP & $136.5 \pm 0.8(739)$ & $138.1 \pm 1.03(436)$ & $137 \pm 1.04(412)$ & $133.9 \pm 2.89(48)$ & $137.3 \pm 2.2(102)$ & $137.6 \pm 3.62(38)$ & $\mathrm{F}(5,1763)=0.5, P=0.77$ \\
\hline TG & $1.72+0.032(1103)$ & $1.7+0.049(652)$ & $1.79+0.056(625)$ & $1.5+0.07(79)$ & $1.92+0.119(144)$ & $1.69+0.083(72)$ & $F(5,2663)=1.8, P=0.11$ \\
\hline TC & $6.03 \pm 0.033(1103)$ & $6.09 \pm 0.045(652)$ & $6.02 \pm 0.044(624)$ & $5.9 \pm 0.103(79)$ & $5.98 \pm 0.102(144)$ & $6.14 \pm 0.124(71)$ & $F(5,2661)=1.0, P=0.40$ \\
\hline TNF & $2.3 \pm 0.07(744)$ & $2.33 \pm 0.1(433)$ & $2.22 \pm 0.09(405)$ & $2.29 \pm 0.26(49)$ & $2.17 \pm 0.18(97)$ & $2.18 \pm 0.3(37)$ & $\mathrm{F}(5,1753)=0.23, P=0.95$ \\
\hline
\end{tabular}

Trait means are shown \pm s.e.m. $(\mathrm{N})$ for untransformed data in relation to TNF locus diplotypes. Significance is shown from ANOVA omnibus tests. Units are as described in Table 1.
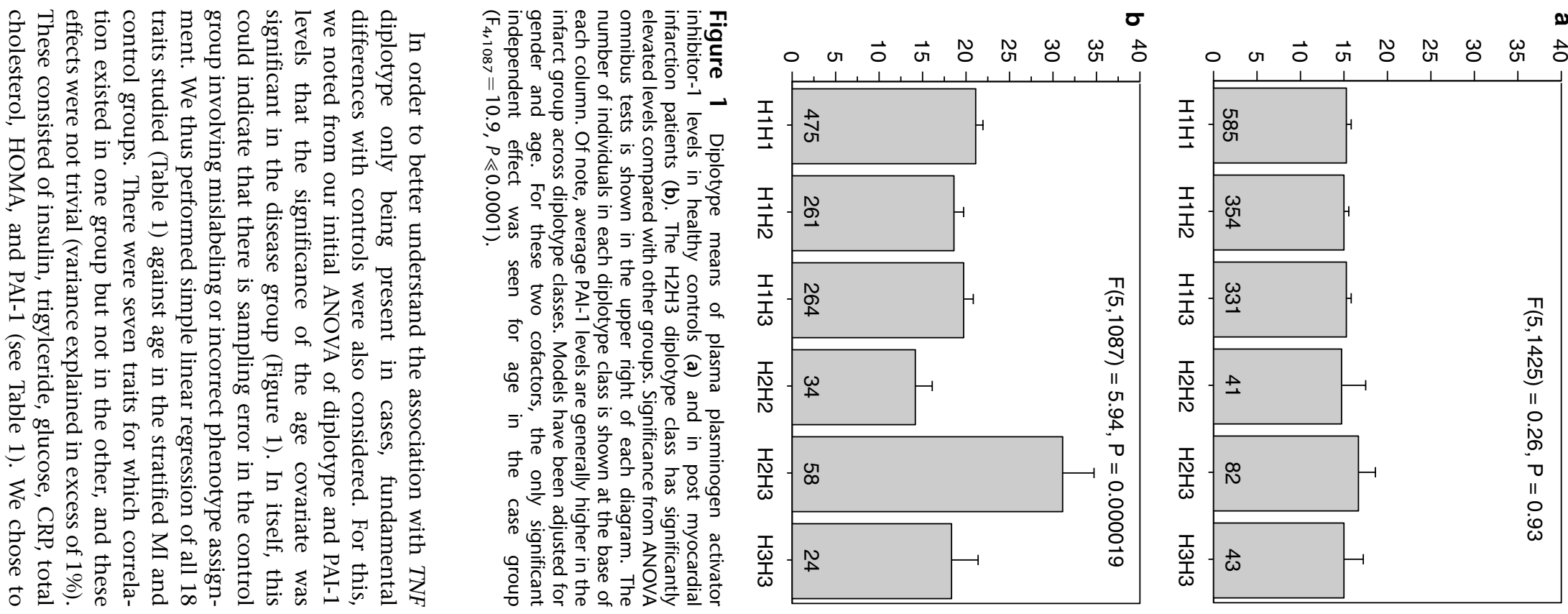

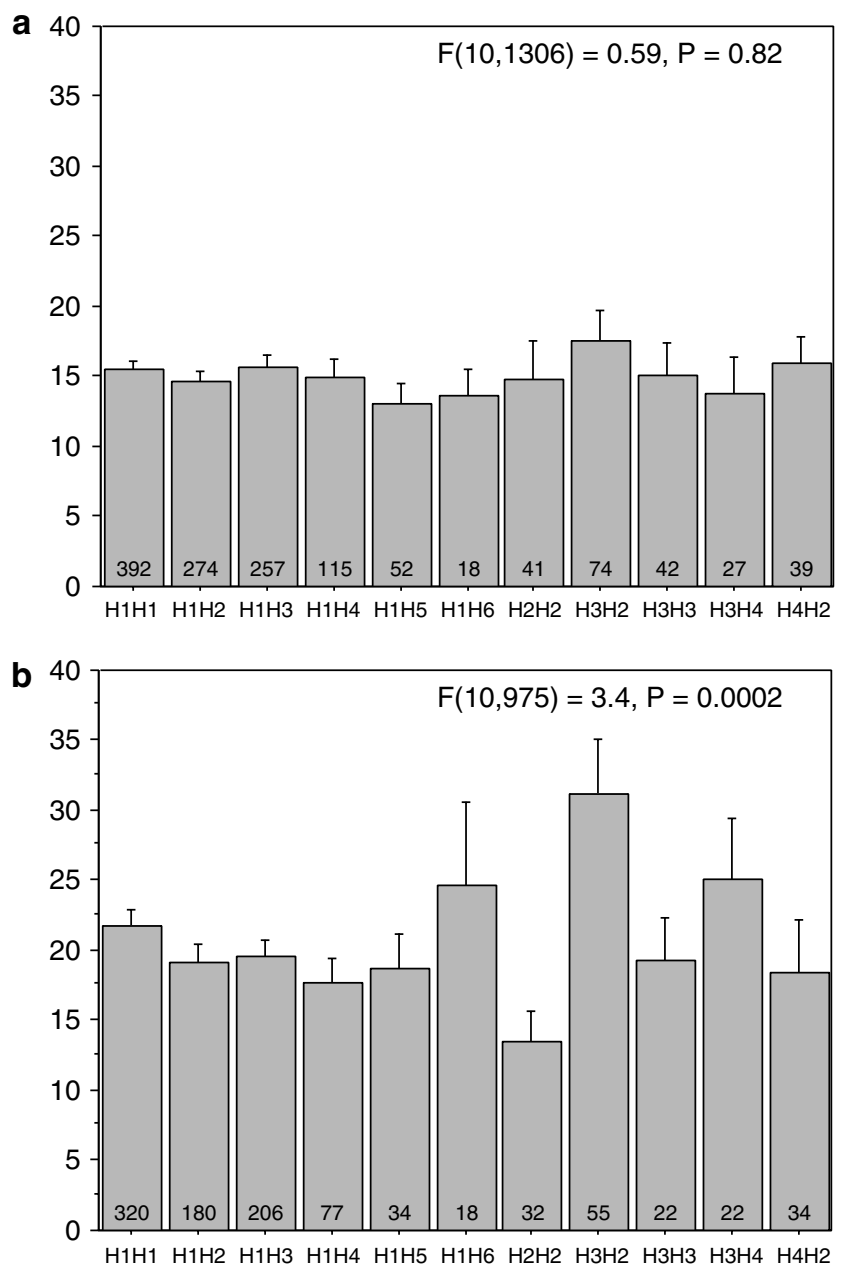

Figure 2 Diplotype means of plasma plasminogen activator inhibitor-1 levels in healthy controls (a) and in post-myocardial infarction patients (b) based upon all five TNF markers. Models have been adjusted for gender and age. Of note, the groups that drive the significance of the model are the same as is seen in Figure 1 (the $\mathrm{H} 2 \mathrm{H} 3$ and $\mathrm{H} 2 \mathrm{H} 2$ classes).

Table 4 Mixed linear ANOVA of five TNF markers and PAl-1 levels

\begin{tabular}{llcl}
\hline & Marker & F Statistic & Singnificance \\
\hline MI Cases & rs1799964 & $\mathrm{F}(2,934)=4.2$ & $P=0.015$ \\
& rs1800630 & $\mathrm{F}(2,934)=2.8$ & $P=0.059$ \\
& rs1799724 & $\mathrm{F}(2,934)=0.2$ & $P=0.82$ \\
& rs1800629 & $\mathrm{F}(2,934)=0.03$ & $P=0.97$ \\
& rs3615525 & $\mathrm{F}(2,934)=8.6$ & $P=0.0002$ \\
Controls & & & \\
& rs1799964 & $\mathrm{F}(2,1181)=1.5$ & $P=0.22$ \\
& rs1800630 & $\mathrm{F}(2,1181)=1.4$ & $P=0.25$ \\
& rs1799724 & $\mathrm{F}(2,1181)=0.9$ & $P=0.41$ \\
& rs1800629 & $\mathrm{F}(2,1181)=0.4$ & $P=0.66$ \\
& rs3615525 & $\mathrm{F}(2,1181)=0.22$ & $P=0.80$ \\
\hline
\end{tabular}

Age and gender have been included as covariates in all analyses. Significant results are highlighted that may indicate that there are 2 or more markers in the region acting independently. highlight three that had the greatest effects (over 2\% variance explained) in their respective groups. Coincidentally these were the traits that emerged as significantly influenced by TNF variation in our initial screen, CRP, HOMA, and PAI-1. We show these relationships in Figure 3.

\section{Discussion}

The TNF locus is one of the more widely studied in human populations, but there still is no unequivocal evidence that variation within it actually impacts any human trait. Our premise for this study has been that, if functional variation in this region actually occurs, and if cardiovascular phenotypes are a reasonable biological target of such variation, then it might be valuable to screen $\mathrm{CHD}$ traits for association in a comprehensive fashion. CHD samples are typically acquired under some of the more strict epidemiological standards for genetic association studies. In addition, CHD study protocols also commonly entail the accumulation of an impressive wealth of ancillary biological phenotypes. This provides an excellent test bed for the kind of experiments illustrated in the present study.

From a screen of 18 CHD-related traits, we provide evidence that $T N F$ polymorphism may be influencing plasma PAI-1 levels. The indicated effect is large, exceeding that of the SERPINE1 4G/5G polymorphism, which is at present the only relatively well-replicated genetic factor affecting circulating levels of PAI- $1 .{ }^{24}$ We also note that although many hypotheses have been addressed, the main finding remains significant after a strict Bonferroni correction for multiple testing. As with any genetic association study, we are cautious about interpreting these results, especially as multiple independent samples are not available for replication purposes. We exhibit the result thus to encourage further independent replication in additional CHD samples that may be able to specifically focus on PAI1. Fortunately, PAI-1 level measurements are fairly common in this field and it should be trivial for other groups to attempt to replicate the effect (or refute it). In addition many groups as judged from the scientific literature have already tested $T N F$-specific polymorphisms for association with primarily MI risk so no additional experimentation may be necessary.

We are also cautious in terms of speculation about the biological plausibility that PAI-1 would be specifically affected by TNF. This is important since linkage disequilibrium could cause association to be seen for loci other than $T N F$. Thus, variability of any of the genes in the region could underlie the observed genetic association, including $T N F$, lymphotoxin-alpha, lymphotoxin-beta, and nuclear factor of kappa light polypeptide all of which have been shown to contribute in some way to inflammatory processes. However, TNF has been demonstrated to be a potent activator of PAI-1, ${ }^{25,26}$ and there is some functional 

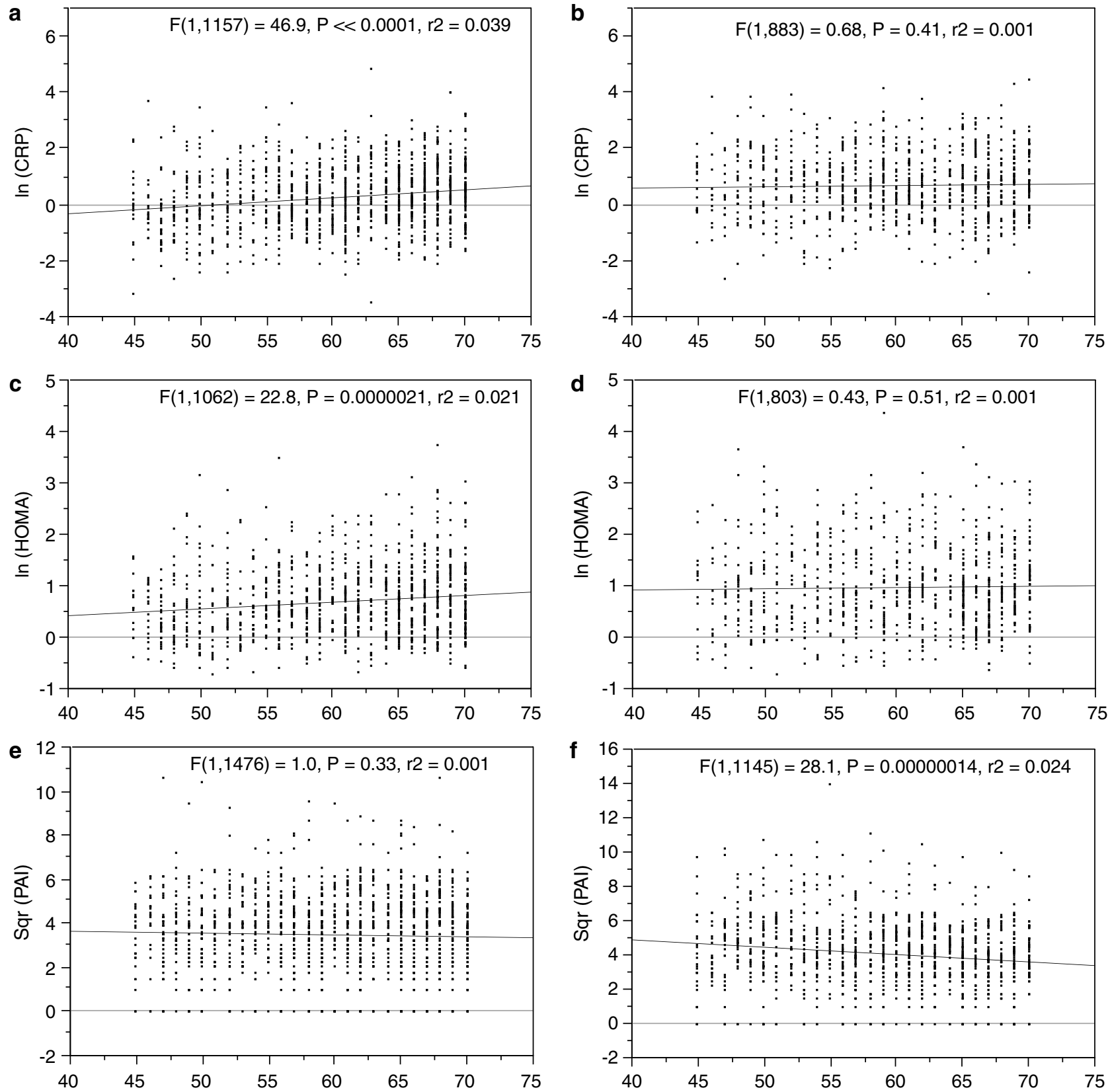

Figure 3 Correlations between age and traits levels for CRP $(\mathbf{a}, \mathbf{b}), \operatorname{HOMA}(\mathbf{c}, \mathbf{d})$ and PAI-1 $(\mathbf{e}, \mathbf{f})$ in MI cases $(\mathbf{a}, \mathbf{c}, \mathbf{e})$ and healthy controls $(\mathbf{b}$, d, $\mathbf{f})$. Trait levels have been transformed for the presentation of this analysis (In for CRP and HOMA and square of PAI-1). This highlights that there are probably fundamental differences in disease and healthy classes that make it important to consider each separately in QTL analyses.

evidence that TNF promoter polymorphisms actually do affect TNF transcription levels. ${ }^{27,28}$ We recognize that mRNA levels may be among the best phenotypes to use to detect functional genetic variability (e.g. Stranger et $a l .{ }^{29}$ ), but these typically are difficult to obtain in large samples, in contrast to the CHD traits reported here. No genetic effect was seen, however, on TNF plasma levels, but this could reflect the biological sample used (plasma) and an effect might be seen in other tissues. Nonetheless, in contrast to, for example, ACE, regulation of TNF plasma levels may not be affected 'detectably' by variation at the TNF locus.

The two main caveats to the observed genetic association are that (a) the genetic model is unusual in that the main effect is mediated by a diplotype hybrid (composed of two unlike haplotypes) and (b) we only observe the effect 
among MI cases. For the first point, similar models have emerged for other convincing genetic association publications (e.g. calpain-10) ${ }^{30}$ One possibility here is that functional alleles occur on different genes in this LD region and that the combination of the two effects the greatest increase in PAI-1 levels. Regardless, this should serve as a warning for further work on the TNF region, that allelic heterogeneity may be present, and that it involves two (or more) different functional sites.

In terms of the association only being evident among cases, we speculate that this may have to do with either the disease process, changes in behavior (stabilization of diet or life-style) following the infarct event, or medication. We observed only the association of SERPINE1 genotypes with PAI-1 levels in the disease population as well, indicating that this group is more in line with what has been observed in other association studies. ${ }^{24}$ We also highlight (Figure 3) correlations that strongly differ between the healthy and the disease groups. This is included primarily to indicate that in retrospective studies, it is appropriate to consider study participants separately according to disease status, especially for CHD-related phenotypes. We acknowledge that this does not offer an explanation for the selective effect of TNF variation on PAI-1 levels. This does however, strengthen our conviction that in this particular sample group-specific effects are not negligible and can exist both when genetic factors and other exposures are considered. Also of note, PAI-1 distributions are very different between cases and controls (see Table 1), consistent with a heightened inflammatory process in MI sufferers. The degree of inflammatory response may be important to consider in future attempts to associate TNF polymorphism with disease end points.

In summary, we present a systematic screen of CHD phenotypes against polymorphism in the TNF locus. Although the predicted association of TNF polymorphism with plasma PAI-1 levels serves as the highlight of this report, we stress that it should primarily be a guide for replication efforts. It should be important to consider this phenotype (perhaps in addition to other metabolic traits) and the models that have emerged here towards the greater goal of proving that functional variation actually occurs in this genomic region and ultimately identifying the molecular character of that variation.

\section{Acknowledgements}

Generous financial support was provided by the Swedish Research Council, King Gustav the Vth and Queen Victoria's Foundation, The Swedish Heart and Lung Foundation, and The Wallenberg Consortium North.

\section{References}

1 Cardon LR, Bell JI: Association study designs for complex diseases. Nat Rev Genet 2001; 2 (2): 91-99.
2 Prince JA, Feuk L, Sawyer SL et al: Lack of replication of association findings in complex disease: an analysis of 15 polymorphisms in prior candidate genes for sporadic Alzheimer's disease. Eur J Hum Genet 2001; 9 (6): 437-444.

3 Ozaki K, Ohnishi Y, Iida A et al: Functional SNPs in the lymphotoxin-alpha gene that are associated with susceptibility to myocardial infarction. Nat Genet 2002; 32 (4): 650-654.

4 Wang WY, Barratt BJ, Clayton DG, Todd JA: Genome-wide association studies: theoretical and practical concerns. Nat Rev Genet 2005; 6 (2): 109-118.

5 Buchanan AV, Weiss KM, Fullerton SM: Dissecting complex disease: the quest for the Philosopher's Stone? Int J Epidemiol 2006; 35 (3): $562-571$.

6 Rigat B, Hubert C, Alhenc-Gelas F, Cambien F, Corvol P, Soubrier F: An insertion/deletion polymorphism in the angiotensin I-converting enzyme gene accounting for half the variance of serum enzyme levels. J Clin Invest 1990; 86 (4): 1343-1346.

7 Cox R, Bouzekri N, Martin S et al: Angiotensin-1-converting enzyme (ACE) plasma concentration is influenced by multiple ACE-linked quantitative trait nucleotides. Hum Mol Genet 2002; 11 (23): 2969-2977.

8 Zabetian CP, Buxbaum SG, Elston RC et al: The structure of linkage disequilibrium at the DBH locus strongly influences the magnitude of association between diallelic markers and plasma dopamine beta-hydroxylase activity. Am J Hum Genet 2003; 72 (6): $1389-1400$.

9 Hennis BC, Boomsma DI, van Boheemen PA et al: An amino acid polymorphism in histidine-rich glycoprotein (HRG) explains 59\% of the variance in plasma HRG levels. Thromb Haemost 1995; 74 (6): 1497-1450.

10 Carlson CS, Aldred SF, Lee PK et al: Polymorphisms within the C-reactive protein (CRP) promoter region are associated with plasma CRP levels. Am J Hum Genet 2005; 77 (1): 64-77.

11 Yamada A, Ichihara S, Murase Y et al: Lack of association of polymorphisms of the lymphotoxin alpha gene with myocardial infarction in Japanese. J Mol Med 2004; 82 (7): 477-483.

12 Bennet AM, van Maarle MC, Hallqvist J et al: Association of TNF-alpha serum levels and TNFA promoter polymorphisms with risk of myocardial infarction. Atherosclerosis 2006; 187 (2): $408-414$.

13 Reuterwall C, Hallqvist J, Ahlbom A et al: Higher relative, but lower absolute risks of myocardial infarction in women than in men: analysis of some major risk factors in the SHEEP study. The SHEEP Study Group. J Intern Med 1999; 246 (2): 161-174.

14 Leander K, Wiman B, Hallqvist J, Falk G, De Faire U: The G-455A polymorphism of the fibrinogen Bbeta-gene relates to plasma fibrinogen in male cases, but does not interact with environmental factors in causing myocardial infarction in either men or women. J Intern Med 2002; 252 (4): 332-341.

15 Leander K, Wiman B, Hallqvist J, Sten-Linder M, de Faire U: PAI-1 level and the PAI-1 4G/5G polymorphism in relation to risk of non-fatal myocardial infarction: results from the Stockholm Heart Epidemiology Program (SHEEP). Thromb Haemost 2003; 89 (6): $1064-1071$

16 Bennet AM, Prince JA, Fei GZ et al: Interleukin-6 serum levels and genotypes influence the risk for myocardial infarction. Atherosclerosis 2003; 171 (2): 359-367.

17 Prince JA, Feuk L, Howell WM et al: Robust and accurate single nucleotide polymorphism genotyping by dynamic allele-specific hybridization (DASH): design criteria and assay validation. Genome Res 2001; 11 (1): 152-162.

18 Stephens M, Smith NJ, Donnelly P: A new statistical method for haplotype reconstruction from population data. Am J Hum Genet 2001; 68 (4): 978-989.

19 Hill W: Estimation of linkage disequilibrium in randomly mating populations. Heredity 1974; 33: 229-239.

20 North K, Howard B, Welty T: Genetic and environmental contributions to cardiovascular disease risk in American Indians: the strong heart family study. Am J Epidemiol 2003; 157: $303-314$. 
21 Broeckel U, Hengstenberg C, Mayer B et al: A comprehensive linkage analysis for myocardial infarction and its related risk factors. Nat Genet 2002; 30: 210-214.

22 Gu HF, Efendic S, Nordman S et al: Quantitative trait loci near the insulin-degrading enzyme (IDE) gene contribute to variation in plasma insulin levels. Diabetes 2004; 53 (8): 2137-2142.

23 Strittmatter WJ, Weisgraber KH, Huang DY et al: Binding of human apolipoprotein $\mathrm{E}$ to synthetic amyloid beta peptide: isoform-specific effects and implications for late-onset Alzheimer disease. Proc Natl Acad Sci USA 1993; 90 (17): 8098-8102.

24 Kathiresan S, Gabriel SB, Yang Q et al: Comprehensive survey of common genetic variation at the plasminogen activator inhibitor-1 locus and relations to circulating plasminogen activator inhibitor-1 levels. Circulation 2005; 112 (12): $1728-1735$.

25 Medcalf RL, Kruithof EK, Schleuning WD: Plasminogen activator inhibitor 1 and 2 are tumor necrosis factor/cachectin-responsive genes. J Exp Med 1988; 168 (2): 751-759.
26 Pandey M, Loskutoff DJ, Samad F: Molecular mechanisms of tumor necrosis factor-alpha-mediated plasminogen activator inhibitor-1 expression in adipocytes. FASEB J 2005; 19 (10): $1317-1319$.

27 Kroeger KM, Carville KS, Abraham LJ: The -308 tumor necrosis factor-alpha promoter polymorphism effects transcription. Mol Immunol 1997; 34 (5): 391-399.

28 Skoog T, van't Hooft FM, Kallin B et al: A common functional polymorphism (C->A substitution at position -863) in the promoter region of the tumour necrosis factor-alpha (TNF-alpha) gene associated with reduced circulating levels of TNF-alpha. Hum Mol Genet 1999; 8 (8): 1443-1449.

29 Stranger BE, Forrest MS, Clark AG et al: Genome-Wide Associations of Gene Expression Variation in Humans. PLoS Genet 2005; 1 (6): e78, [E-pub ahead of print].

30 Horikawa Y, Oda N, Cox NJ et al: Genetic variation in the gene encoding calpain-10 is associated with type 2 diabetes mellitus. Nat Genet 2000; 26 (2): 163-175. 